\title{
THE BOUNDED LINEAR OPERATORS THAT COMMUTE WITH THE BERNSTEIN OPERATORS
}

\author{
BY A. G. KONHEIM AND T. J. RIVLIN
}

Communicated by R. C. Buck, August 10, 1967

Let $C[0,1]$ denote the linear space of real-valued continuous functions on $[0,1]$ normed by

$$
\|f\|=\max _{0 \leqq x \leqq 1}|f(x)|
$$

and $P_{n}$ the subspace of $C[0,1]$ consisting of the polynomials of degree $\leqq n$. For each $n(n=0,1,2, \cdots)$ we denote by $B_{n}$ the operator

$$
B_{n}: C[0,1] \rightarrow P_{n}
$$

defined by

$$
\begin{aligned}
& \left(B_{n} f\right)(x)=\sum_{k=0}^{n} f(k / n)\left(\begin{array}{l}
n \\
k
\end{array}\right) x^{k}(1-x)^{n-k} \quad(n \geqq 1), \\
& \left(B_{0} f\right)(x)=f(0) .
\end{aligned}
$$

We call $B_{n}$ the Bernstein operator of order $n$ and $B_{n} f$ the nth Bernstein polynomial of $f$. The purpose of this note is to characterize those bounded linear operators $T$

$$
T: C[0,1] \rightarrow C[0,1]
$$

which satisfy

$$
T B_{n}=B_{n} T \quad(n=0,1,2, \cdots)
$$

on $C[0,1]$. We observe that it is sufficient to require (1) to hold on $P=\mathrm{U}_{n} P_{n}$ since $P$ is dense in $C[0,1]$.

LEMMA 1. (a) $B_{n} f=0$ if and only if

$$
\begin{aligned}
f \in N_{n} & =\{g \in C[0,1]: g(k / n)=0, \quad 0 \leqq k \leqq n\}, \quad(n \geqq 1), \\
\left(N_{0}\right. & =\{g \in C[0,1]: g(0)=0\}) .
\end{aligned}
$$

(b) $B_{n}$ is onto $P_{n}$.

Lemma 2. If $T$ satisfies (1) on $P$ then

(a) $T: P_{n} \rightarrow P_{n}$;

(b) $f \in N_{n}$ implies $T f \in N_{n}$.

Proof. $B_{n} T f \in P_{n}$ and hence $T B_{n} f \in P_{n}$. Since $B_{n}$ is onto $P_{n}$, (a) is 
established. Next, suppose that $f \in N_{n}$. Then $T B_{n} f=0$ and hence $B_{n} T f=0$, which implies that $T f \in N_{n}$.

Definition. Let $\left\{p_{k}: k=0,1,2, \cdots\right\}$ be the family of polynomials

$$
\begin{aligned}
p_{k}(x) & =1 \quad \text { if } k=0 \\
& =x \quad \text { if } k=1 \\
& =\prod_{i=0}^{k-1}\left(x-\frac{i}{k-1}\right) \quad \text { if } 2 \leqq k<\infty .
\end{aligned}
$$

Lemma 3. (a) The $\left\{p_{k}: k=0,1,2, \cdots\right\}$ are linearly independent and each $p \in P_{n}$ admits a unique expansion $p=a_{0} p_{0}+a_{1} p_{1}+\cdots+a_{n} p_{n}$.

(b) If $B_{n} p_{k}=\sum_{s=0}^{n} C_{s}(n, k) p_{s}$ then

(i) $C_{s}(n, k)=0$ if $s \neq \equiv(\bmod 2)$,

(ii) $C_{0}(n, k)=C_{1}(n, k)=0$ if $2 \leqq k<\infty$,

(iii) $C_{s}(n, k)=0$ if $s>k$.

Proof. (a) is obvious. To prove $(\mathrm{b}, \mathrm{i})$ we observe that for $k \neq 1$

$$
(-1)^{k}\left(B_{n} p_{k}\right)(x)=\left(B_{n} p_{k}\right)(1-x)=\sum_{s=0}^{n} C_{s}(n, k)(-1)^{s} p_{s}(x)+C_{1}(n, k) .
$$

For $2 \leqq k<\infty$ we have $\left(B_{n} p_{k}\right)(0)=p_{k}(0)=0$ so that $C_{0}(n, k)=0$. In addition $\left(B_{n} p_{k}\right)(1)=p_{k}(1)=0(2 \leqq k<\infty)$ from which we may conclude that $C_{1}(n, k)=0(2 \leqq k<\infty)$. Finally, the image of $P_{k}$ under $B_{n}$ is just $P_{k}$ for $k \leqq n$, and this gives (b, iii).

Definition. Define the operators $U_{0}, U_{1}, U$ and $\tilde{U}$ by

$$
\begin{aligned}
\left(U_{0} f\right)(x) & =f(0), \quad\left(U_{1} f\right)(x)=(f(1)-f(0)) x, \\
(U f)(x) & =\frac{1}{2}(f(x)+f(1-x)), \quad \tilde{U}=I-U .
\end{aligned}
$$

LEMMA 4. For each $n, n=0,1,2, \cdots$,

$$
\begin{aligned}
U_{0} B_{n} & =B_{n} U_{0}, & U_{1} B_{n}=B_{n} U_{1}, \\
U B_{n} & =B_{n} U, & \tilde{U} B_{n}=B_{n} \tilde{U} .
\end{aligned}
$$

Proof. $\quad\left(U_{0} B_{n} f\right)(x)=\left(B_{n} f\right)(0)=f(0)=\left(B_{n} U_{0} f\right)(x) . \quad\left(U_{1} B_{n} f\right)(x)$ $=\left(\left(B_{n} f\right)(1)-\left(B_{n} f\right)(0)\right) x=(f(1)-f(0)) x=\left(B_{n} U_{1} f\right)(x)$. Finally

$$
\begin{aligned}
\left(B_{n} U f\right)(x) & =\sum_{k=0}^{n} \frac{f(k / n)+f(1-k / n)}{2}\left(\begin{array}{l}
n \\
k
\end{array}\right) x^{k}(1-x)^{n-k} \\
& =\sum_{k=0}^{n} f(k / n)\left(\begin{array}{l}
n \\
k
\end{array}\right) \frac{x^{k}(1-x)^{n-k}+(1-x)^{k} x^{n-k}}{2} \\
& =\left(U B_{n} f\right)(x) .
\end{aligned}
$$


The result for $\tilde{U}$ now follows, since $\tilde{U}=I-U$.

Theorem. A necessary and sufficient condition that a bounded linear operator $T: C[0,1] \rightarrow C[0,1]$ satisfy (1) is that

(2) $T=a_{0} U_{0}+a_{1} U_{1}+a U\left(I-U_{0}-U_{1}\right)+\tilde{a} \tilde{U}\left(I-U_{0}-U_{1}\right)$.

Proof. (i) By Lemma 4 it follows that any operator of the form (2) satisfies (1).

(ii) By Lemma 2(a) we have $T p_{0}=\sigma_{0} p_{0}$ and by Lemma 2(a, b) $T p_{k}=\sigma_{k} p_{k}$ for $1 \leqq k<\infty$. By Lemma $3(\mathrm{a})$ this determines $T$ on $P$. It suffices to determine the $\left\{\sigma_{k}\right\}$ such that $T B_{n} p_{k}=B_{n} T p_{k}$ for all $k$ and $n$. Now

$$
\begin{array}{ll}
T B_{n} p_{0}=T p_{0}=\sigma_{0} p_{0}=B_{n} T p_{0} & (n=0,1,2, \cdots), \\
T B_{n} p_{1}=T p_{1}=\sigma_{1} p_{1}=B_{n} T p_{1} & (n=1,2, \cdots),
\end{array}
$$

while $T B_{0} p_{1}=0=B_{0} T p_{1}$. Thus $\sigma_{0}$ and $\sigma_{1}$ may be chosen arbitrarily. Henceforth assume that $2 \leqq k<\infty$. Then

$$
T B_{n} p_{k}=\sum_{s=2}^{n} C_{s}(n, k) \sigma_{s} p_{s}
$$

while

$$
B_{n} T p_{k}=\sum_{s=2}^{n} C_{s}(n, k) \sigma_{k} p_{s}
$$

so that $C_{s}(n, k)\left(\sigma_{s}-\sigma_{k}\right)=0(2 \leqq s \leqq n, 2 \leqq k<\infty, n=0,1,2, \cdots)$. If we take $n=2$, then $B_{n} p_{k} \neq 0$ for $k \equiv 0(\bmod 2)$ and hence $C_{2}(2, k) \neq 0$ for $k \equiv 0(\bmod 2)$. Thus $\sigma_{k}=\sigma_{2}$ for $k \equiv 0(\bmod 2)$. Assume next that $\sigma_{3}=\sigma_{5}=\cdots=\sigma_{2 j+1}$. Then by Lemma 3(b, i)

$$
B_{2 j+1} p_{2 j+3}=\sum_{\substack{0 \leq s \leq 2 j+1 ; \\ s=1(\bmod 2)}} C_{s}(2 j+1,2 j+3) p_{s} .
$$

Since $B_{2 j+1} p_{2 j+3} \neq 0$ there exists a $j_{0}, 0<j_{0} \leqq j$ such that $C_{2 j_{0+1}}(2 j+1,2 j+3) \neq 0$ and this implies that $\sigma_{2 j+3}=\sigma_{2 j+1}$. Hence

$$
\begin{array}{ll}
\sigma_{k}=\sigma_{2} & \text { if } k \equiv 0(\bmod 2) 2 \leqq k<\infty, \\
\sigma_{k}=\sigma_{3} & \text { if } k \equiv 1(\bmod 2) 2 \leqq k<\infty,
\end{array}
$$

so that if $p=\sum_{s=0}^{n} b_{s} p_{s}$ then

$$
T p=\sigma_{0} b_{0} p_{0}+\sigma_{1} b_{1} p_{1}+\sigma_{2} \sum_{2 \leq s \leq n ; s=0(\bmod 2)} b_{s} p_{s}+\sigma_{3} \sum_{2 \leq s \leq n ; s=1(\bmod 2)} b_{s} p_{s} .
$$

Finally $b_{0}=p(0)$ and $b_{0}+b_{1}=p(1)$ and thus

$$
T p=\sigma_{0} U_{0} p+\sigma_{1} U_{1} p+\sigma_{2} U\left(I-U_{0}-U_{1}\right) p+\sigma_{3} \tilde{U}\left(I-U_{0}-U_{1}\right) p
$$


satisfies (2) on $P$. But $P$ is dense in $C[0,1]$ and hence $T$ satisfies (2) on $C=[0,1]$.

REMARK. The same methods lead to a somewhat more general result. Suppose that for each $n, n=0,1,2, \ldots X_{n}$ denotes the set $\left\{x_{0}^{(n)}, x_{1}^{(n)}, \cdots, x_{n}^{(n)}\right\}$ of distinct points on $[0,1]$, with the properties that for $n \geqq 3$ there exists some $i$ such that $X_{i} \Phi X_{n}$ and that $x_{0}^{(n)}=0(n=0,1,2, \cdots)$. Let $B_{k}(k=0,1,2, \cdots)$ be a bounded linear operator $B_{k}: C[0,1] \rightarrow P_{k}(k=0,1,2, \cdots)$ satisfying:

(1) $B_{k} 1=1(k=0,1,2, \cdots), B_{k} x=x(k=1,2, \cdots), B_{0} f=f(0)$.

(2) $B_{k f} f=0$ if and only if

$$
f \in N_{k}=\left\{g \in C[0,1]: g\left(x_{k}^{(j)}\right)=0,0 \leqq j \leqq k\right\} .
$$

(3) $B_{k} S=S B_{k}(k=0,1,2, \cdots)$ where $(S f)(x)=f(1-x)$.

Then the Theorem holds with an identical proof which we omit. In addition to the Bernstein operators, the (Lagrange) interpolation operators, $L_{k}$, defined by

$$
\left(L_{k} f\right)(x)=\sum_{j=0}^{k} f\left(x_{j}^{(k)}\right) \prod_{\substack{i=0 ; \\ i \neq j}}^{k} \frac{x-x_{i}^{(k)}}{x_{i}^{(k)}-x_{i}^{(k)}}
$$

are included in the more general result, provided that the interpolating sets $X_{k}$ are invariant under the transformation $x \rightarrow 1-x$.

We want to thank G. Rota for bringing this problem to our attention.

ibM Watson Research Center, Yorktown Heights, New York 